\title{
Estudio de la respuesta a ejercicios de resistencia de tipo aeróbico en función de la tipología del pie en la población infantil
}

\author{
Study of the response to aerobic resistance exercises based on the type of foot in the child \\ population
}

\author{
María de los Ángeles Gómez-Benítez ${ }^{1}$, Javier Ramos-Ortega² y Andrea Gómez-Benítez ${ }^{3}$ \\ 'Práctiva privada. Sevilla, España. ${ }^{2}$ Departamento de Podología, Universidad de Sevilla. Sevilla, España. ${ }^{3}$ Hospital Comarcal La Línea de la \\ Concepción. Cádiz, España.
}

Palabras clave:

Pronación, niños, pies pronados, ejercicio aeróbico, ejercicio de resistencia.

\section{Keywords:}

Pronation, children, pronated feet, aerobic exersice, resistance exercises.
Recibido: 29-01-2020 Aceptado: 16-06-2020

\section{Resumen}

Introducción: El objetivo principal de este trabajo fue comprobar si los niños con pies pronados obtienen resultados diferentes en ejercicios de resistencia de tipo aeróbicos respecto a los niños que presentan una tipología de pies neutra según el Foot Posture Index.

Pacientes y métodos: Se trata de un estudio trasversal de tipo descriptivo y observacional. Ciento cinco niños, de edades comprendidas entre 10 y 12 años (10.46 \pm 0.785), fueron reclutados y divididos en dos grupos: 63 formaron el grupo control o pies neutros y 42 el grupo casos o pies pronados; 51 fueron niños y 54 niñas. Se seleccionaron cinco ejercicios de resistencia de tipo aeróbico para evaluar el rendimiento motor en ambos tipos de pies, mediante un circuito previamente establecido: salto a la comba con desplazamiento, desplazamientos a $8 \mathrm{~m}$ de distancia, alternancia piernas en banco sueco, minicircuito en zigzag y salto con pies juntos. Se realizaron tres series de ejercicios, donde existió un periodo de descanso de 3-5 minutos entre cada serie. En cada estación se fue calculando el número de repeticiones que realizaba el niño.

Resultados: Los resultados obtenidos de nuestro estudio indicaron que ambos grupos obtuvieron resultados muy similares en las pruebas físicas de resistencia $(p>0.05)$. La muestra fue homogénea en cuanto a sexo, edad e IMC $(p>0.05)$ y respecto a la variable "veces a la semana que realizaban deporte los niños" ( $p>0.05)$.

Conclusiones: Según los datos obtenidos en el presente estudio, no se han encontrado diferencias significativas en el rendimiento motor entre niños con pies pronadores y niños con pies neutros, en los 5 ejercicios de resistencia evaluados en el presente estudio, por lo que no se puede descartar la hipótesis nula de la investigación.

\section{Abstract}

Introduction: The main objective of this work was to verify whether children with pronator feet obtain different results in aerobic resistance exercises compared to children who have a neutral foot typology according to the Foot Posture Index.

Patients and methods: This is an cross-sectional study of type observational and descriptive. One hundred and five children, aged between 10 and 12 years $(10.46 \pm 0.785)$ were recruited and divided into two groups: 63 formed the control group or neutral feet and 42 the case group or pronator feet. Of which 51 were boys and 54 girls. Five resistance exercises of aerobic type were selected to evaluate the motor performance in both types of feet, by means of a previously established circuit: jump rope with displacement, displacements at $8 \mathrm{~m}$ distance, alternation legs in Swedish bench, mini-circuit in zig-zag, jump with feet together. Three series of exercises were carried out, where there was a 3-5 minutes rest period between each series. In each station the number of repetitions the child performed was calculated.

Results: The results obtained from our study indicate that both groups obtained very similar results in physical resistance tests $(p>0.05)$. The sample was homogeneous in terms of sex, age and BMI ( $p>0.05)$ and with respect to the variable "times a week children perform sports" ( $p>0.05)$.

Conclusions: These results suggest that children with neutral feet did not achieve better motor performance compared to children with pronated feet in the 5 resistance exercises evaluated in our study. 


\section{INTRODUCCIÓN}

La práctica de actividad física regularmente es importante a la hora de prevenir hábitos sendentarios que puedan desencadenar problemas futuros de salud. Es por eso que hoy en día se intenta fomentar el ejercicio desde la niñez. El pie, como primer eslabón en la cadena, se considera de especial importancia en el deporte y en cualquier tipo de actividad física, constituyendo la base de apoyo para el resto de movimientos del cuerpo.

Biomecánicamente, es razonable considerar que pequeñas alteraciones en la estructura o alineación del pie y tobillo podrían influir en el rendimiento deportivo de la actividad realizada, repercutiendo en el resto del cuerpo y condicionando la elección de los sujetos para un determinado deporte o simplemente para su vida diaria. Hoy en día, dado el impacto que supone el deporte en nuestra sociedad, el interés por conocer los mecanismos biomecánicos que inducen a fatiga muscular, disminución del rendimiento y lesión es un área que debe estar en constante estudio para clínicos e investigadores.

Algunos autores coinciden en que en el pie pronador existe una alteración de los ejes de movimiento, lo que podría favorecer a que los músculos de la pierna y del pie tiendan a la fatiga con facilidad ${ }^{1-6}$ siendo aún mayor en la práctica deportiva ${ }^{4}$. La controversia entre los diferentes autores es relevante ${ }^{7-17}$, no existiendo un consenso de si el tipo de pie repercute en el rendimiento motor y si específicamente el pie pronador presenta algún tipo de desventaja respecto al pie neutro en el deporte. Este es un dato importante, ya que la prevención y mejora de las alteraciones biomecánicas en etapas infantiles, mediante soportes plantares, sería un punto destacable en este aspecto.

Aunque se sabe que los diferentes tipos de pies pueden influir en la cinemática, la presión plantar ${ }^{16,18,19}$, huella plantar $^{9,13,15}$, postura del pie ${ }^{17}$ y electromiografía (EMG) ${ }^{20}$ de ciertas actividades deportivas, no se han realizado estudios, según el conocimiento de los autores, que informen de cómo los ejercicios de resistencia podrían influir en niños con 10-12 años con pies pronados y con pies neutros, según los define el Foot Posture Index ${ }^{21}$.

El objetivo principal de este trabajo fue comprobar si los niños con pies pronados obtenían resultados diferentes en ejercicios de resistencia de tipo aeróbicos respecto a los niños que presentaban una tipología de pies neutra.

\section{PACIENTES Y MÉTODOS}

Según las características del presente trabajo, se trata de un estudio trasversal de tipo observacional y descriptivo. Para su realización, se sometió el trabajo a evaluación por el comité ético de investigación de los Hospitales Universitarios Virgen Macarena-Virgen del Rocío, obteniendo una valoración favorable.

\section{Población estudio}

Los/as niños/as del estudio estaban matriculados en $5 .^{\circ} \mathrm{y}$ $6 .^{\circ}$ de primaria de dos centros educativos de la localidad de Jimena de la Frontera, provincia de Cádiz, Andalucía, España. Los dos centros docentes son públicos y contaban con instalaciones adecuadas, como una pista polideportiva exterior y un salón interior habilitado para actividades deportivas.

La recogida de datos se llevó a cabo durante los años 2014, 2015 y 2016, siempre en horario de mañana, previo consentimiento informado de los padres.

Los niños que participaron en el estudio debían cumplir rigurosamente con los criterios de selección establecidos. Se incluyeron aquellos que presentaban un índice de masa corporal (IMC) equivalente a normo-peso, es decir un percentil entre 5 y 85 según el porcentaje del IMC respecto la edad $^{22,23}$, y una tipología de pie pronador o de pie neutro según el FPI-6 ${ }^{21}$.

Se excluyeron a todos aquellos que se encontraban en tratamiento ortopodológico en el momento del estudio, aquellos que no pudieron completar las pruebas físicas establecidas o habían experimentado algún tipo de cirugía osteoarticular, traumatismo o enfermedades osteoarticulares degenerativas en los pies o miembros inferiores (MMII) y aquellos que presentaban cada uno de sus pies en una categorización diferente según el FPI-6 ${ }^{21}$.

\section{Variables estudiadas}

A continuación se enumeran las variables utilizadas en esta investigación, diferenciando entre variables cualitativas (sexo, con la categoría niño y niña) y variables cuantitativas: edad cuantificada en años, IMC [peso/ altura] cuantificado en $\mathrm{kg} / \mathrm{m}^{2}$, Foot Posture Index, veces a la semana que realizaban deporte los niños y las pruebas físicas (salto a la comba con desplazamiento [3 series], desplazamientos a $8 \mathrm{~m}$ de distancia [3 series], alternancia piernas en banco sueco [3 series], minicircuito en zigzag [3 series], salto con pies juntos [3 series]).

\section{Intervención}

Los datos fueron extraídos por un único examinador (M.A.G.B.) con el fin de evitar el posible error interpersonal. El protocolo llevado a cabo siguió siempre el mismo orden que se expresa a continuación.

En primer lugar se tomaron las medidas antropométricas mediante un peso y un tallímetro para posteriormente calcular el IMC del sujeto, el cual determinaba en qué percentil se encontraba el niño y si debía incluirse o no en el estudio, mediante la fórmula IMC $=$ peso $(\mathrm{kg}) /$ altura $(\mathrm{m})^{2}$. Si el valor obtenido de la fórmula correspondía a un percentil comprendido entre 3 y 90 , equivalente a normo-peso, el sujeto se incluía en el estudio.

Luego se valoró la postura del pie mediante el FPI-6 ${ }^{21}$ para clasificarlos en dos grupos: pies pronados o pies neutros, 
según la tipología de pies. Se incorporaron en el grupo control aquellos individuos con valores de $0 \mathrm{a}+5$ (correspondientes a pie neutro) y en el grupo casos o pies pronados valores de +6 $\mathrm{a}+9$ (pronado) y de $+10 \mathrm{a}+12$ (altamente pronado)

Seguidamente se valoró el tipo de calzado que llevaba el niño, pues era importante que no llevara elementos que los hicieran ser zapatillas "pronadoras" o "supinadoras", que tuviera características de zapato saludable y apto para una actividad deportiva. Aquellos que no lo llevaban en el momento del estudio, realizaron las pruebas físicas otro día diferente, y seguidamente se preguntó a los niños cuántas veces a la semana realizaban deporte, anotándose en nuestra hoja de recogida de datos.

La actividad deportiva que se llevó a cabo fueron ejercicios de resistencia de tipo aeróbicos. Los ejercicios consistían en 6 estaciones, donde cada niño permaneció 30 segundos. En cada estación se calculó el número de repeticiones que realizaba el niño. Se realizaron 3 series de ejercicios donde existió un periodo de descanso de 3-5 minutos entre cada serie. El colaborador que contabilizaba las repeticiones estuvo cegado respecto al tipo de pie del niño/a y el investigador que clasificó los grupos (M. A. G. B.) fue diferente al que contabilizó las pruebas físicas.

Las estaciones realizadas fueron: carrera suave para iniciar los ejercicios con mejor aptitud física, salto a la comba con desplazamiento, desplazamientos tocando líneas a $8 \mathrm{~m}$ de distancia, alternancia de piernas en banco sueco, minicircuito en zigzag y salto con los pies juntos.

\section{Análisis estadístico}

Para realizar el análisis estadístico se utilizó el paquete estadístico SPSS Statistics 22. Para el análisis descriptivo de las variables se han calculado los valores medios, la desviación típica (DT), el mínimo y el máximo.
Se realizó el test de Kolmogorov-Smirnov para comprobar si los datos seguían una distribución normal. Fruto de esta prueba se decidió que se utilizaría la prueba U de Mann-Whitney para las comparaciones de las variables salto a la comba con desplazamiento, desplazamientos a 8 metros de distancia, circuito en zigzag y salto con los pies juntos. Para las variables alternancia piernas en banco sueco, se utilizó la t de Student para muestras independientes. Se ha considerado estadísticamente significativo, siempre que el valor de $p$ haya sido menor que 0.05 .

\section{RESULTADOS}

La muestra de estudio se compuso de 105 participantes, de los cuales 51 fueron niños y 54 niñas, de edades comprendidas entre los 10 y 12 años (10.46 \pm 0.785$)$. De los 105 niños/as, 63 formaron el grupo control o grupo de pies neutros, de los cuales 35 fueron niños y 28 niñas, y 42 el grupo casos o grupo de pies pronados, de los cuales 16 fueron niños y 26 niñas. La muestra fue homogénea en cuanto a sexo, edad e IMC $(p>0.05)$ y respecto a la variable "veces a la semana que realizaban deporte los niños" ( $p=0.105)$.

A nivel descriptivo se expresaron los valores medios, la desviación típica y el máximo y mínimo de las cinco variables pertenecientes a las pruebas físicas llevadas a cabo en el estudio, para el grupo casos y para el grupo control (Tabla I).

Se compararon los valores centrales de las tres repeticiones de una misma prueba física para comprobar si había diferencias significativas en el número de repeticiones que realizaban los niños del grupo pies neutros con los del grupo pies pronados. Los resultados mostraron valores no estadísticamente significativos, es decir, no se encontraron diferencias en los resultados de las pruebas físicas entre grupos (Tabla II).

\begin{tabular}{|c|c|c|c|c|c|c|}
\hline & & $\begin{array}{l}\text { Salto comba } \\
\text { (núm. saltos) }\end{array}$ & $\begin{array}{l}\text { Desplazamiento } \\
\text { en } 8 \mathrm{~m} \\
\text { (repeticiones) }\end{array}$ & $\begin{array}{l}\text { Banco sueco } \\
\text { (núm. saltos) }\end{array}$ & $\begin{array}{l}\text { Circuito zigzag } \\
\text { (repeticiones) }\end{array}$ & $\begin{array}{l}\text { Salto con pies } \\
\text { juntos } \\
\text { (núm. saltos) }\end{array}$ \\
\hline \multirow{3}{*}{ Grupo Control } & Media \pm DT & $28.70 \pm 8.59$ & $9.30 \pm 1.20$ & $52.11 \pm 12.45$ & $3.40 \pm 0.74$ & $63.78 \pm 10.41$ \\
\hline & Mín. & 8 & 7 & 23 & 2 & 33 \\
\hline & Máx. & 48 & 14 & 83 & 5 & 100 \\
\hline \multirow{3}{*}{ Grupo Casos } & Media \pm DT & $26.24 \pm 7.50$ & $9.02 \pm 1.31$ & $47.9 \pm 12.67$ & $3.36 \pm 0.75$ & $62.36 \pm 9.25$ \\
\hline & Mín. & 7 & 7 & 22 & 2 & 45 \\
\hline & Máx. & 41 & 12 & 72 & 5 & 87 \\
\hline
\end{tabular}

Núm: número. DT: desviación estándar. 
Tabla II. Significación estadística de las pruebas físicas.

\begin{tabular}{ll} 
Pruebas físicas & Significación $p$ \\
\hline Salto comba & 0.171 \\
\hline Desplazamiento a 8 $\mathrm{m}$ & 0.239 \\
\hline Alter. piernas banco sueco & 0.095 \\
\hline Circuito zigzag & 0.825 \\
\hline Salto con pies juntos & 0.332 \\
\hline
\end{tabular}

\section{DISCUSIÓN}

Los resultados obtenidos de nuestro estudio indicaron que ambos grupos obtuvieron resultados muy similares en las pruebas físicas de resistencia. Sin embargo, tenemos que reconocer que sí hubo diferencias descriptivas en los valores obtenidos en cada una de las pruebas físicas entre el grupo casos y el grupo control. Es decir, los niños/as con pies pronados obtuvieron menos repeticiones en las pruebas físicas respecto a los niños con pies neutros. Aunque el resultado no fue estadísticamente significativo en ninguno de los cinco test realizados, en dos de ellos, salto a la comba con desplazamiento y saltos en banco sueco, los resultados se aproximaron a la significación.

Conforme a lo mencionado en diversos artículos que valoraban los tipos de pies mediante diversos ejercicios, observamos que sometían a los individuos a la combinación de ejercicios de fuerza, resistencia, velocidad y flexibilidad, obteniendo en su mayoría resultados no significativos $7,8,10,12,24,25$.

Tanto la fuerza como la resistencia, la velocidad y la flexibilidad son capacidades físicas básicas que se van desarrollando a lo largo del crecimiento del niño ${ }^{26}$. Según Mora ${ }^{26}$, la resistencia aeróbica es la capacidad física de realizar un esfuerzo disponiendo durante toda la actividad de oxígeno suficiente para la oxidación de los sustratos energéticos (glucosa y ácidos grasos) que serán necesarios para la contracción muscular. La evolución de esta capacidad físico-motriz va ligada al crecimiento y transformaciones del aparato cardiovascular. Así, el aumento del volumen del corazón durante esta fase de la vida infantil incrementará el volumen de oxígeno $\left(\mathrm{Vo}^{2}\right)$ máximo. El desarrollo de esta capacidad comienza a partir de los 6 años y experimenta su progreso máximo a los 10-11 años, permaneciendo estable hasta los 15 años $^{26}$. En este caso, consideramos que los 10-12 años era la edad de estudio óptima, ya que permanecería estable hasta los 15 años.

Fue difícil comparar nuestro trabajo con los encontrados, pues todos estudiaban el rendimiento de los diferentes tipos de pie en diferentes actividades físicas y pruebas motrices, y nosotros solo incluimos la capacidad física de resistencia. Sin embargo, nuestros hallazgos coinciden a groso modo con lo que la mayoría de los autores afirman. Kaufman y cols. ${ }^{25}$ valoraron si el tipo de pie era un factor influyente en la actividad física en 50 sujetos con pies planos y 50 con pies neutros tras realizar una serie de ejercicios físicos de agilidad, equilibrio y velocidad. Sus resultados mostraron que el rendimiento físico en los dos grupos del estudio era similar y no dependía de la morfología del pie. De igual manera, Arévalo en $2013^{12}$ realizó un estudio muy parecido donde incluía 187 individuos de 10-12 años para estudiar si algún tipo de pie (normal, cavo, plano) tenía mayor rendimiento en pruebas motrices de saltos de longitud, triples saltos, equilibrios y circuitos de agilidad. En sus conclusiones señaló que el tipo de pie no repercutía en el rendimiento motor en ese tipo de pruebas y ningún tipo de pie fue mejor que otro, a excepción de los pies cavos que obtuvieron mejores resultados.

Tudor y cols., en $2009^{10}$, realizaron cuatro grupos en función del grado de aplanamiento del arco y los sometieron a una serie de pruebas físicas, salto, velocidad, equilibrio y otras como flexión de los dedos y ponerse de puntillas. No encontraron correlación entre la altura del arco y las 17 habilidades motoras realizadas. De igual manera, afirmaron que no existía desventaja en el rendimiento deportivo tras comparar los grupos de pies extremos (arcos muy altos y arcos muy bajos). Kumala y cols., en $2019^{7}$, estudiaron las diferencias en el rendimiento físico en atletas de 14-17 años con pies planos y pies neutros mediante ejercicios de fuerza, equilibrio y velocidad. No se encontraron diferencias en el rendimiento físico de cada una de las pruebas en ambos grupos. Autores como Roohi y cols. ${ }^{8}$ sometieron a un grupo formado por pies pronados y a otro grupo por pies neutros a pruebas de velocidad, agilidad y valoraciones estáticas y dinámicas. Tan solo encontraron diferencias entre los grupos respecto a las pruebas de agilidad y equilibrio estáti$\mathrm{co}$, no encontrando diferencias en las pruebas de velocidad y equilibrio dinámico, resultados difíciles de comparar con los de nuestro estudio, pues solo incluimos la capacidad física básica de resistencia a diferencia de estos, que incluyen varias.

Sin embargo, Lin y cols. ${ }^{27}$ discreparon con los resultados de todos estos autores, pues afirmaron haber obtenido puntuaciones peores en los pies planos moderados y severos respecto a los neutros y cavos en diferentes tareas atléticas.

Estimamos necesario aclarar que una de las limitaciones más evidentes que posee nuestro estudio se refiere a la muestra estudiada; podríamos decir que al ser relativamente pequeña, no es lo suficientemente representativa como para que los resultados fuesen más concluyentes. Este aspecto está directamente relacionado con la potencia: es posible que los resultados hayan sido negativos por la baja potencia del estudio. Por otro lado, la escasa información sobre estudios que valoren los diferentes tipos de pie exclusivamente mediante pruebas de resistencia podría haber influido en que las estaciones llevadas a cabo o los intervalos de tiempo establecidos no fueran las más apropiadas para obtener resultados concluyentes.

En conclusión, los hallazgos del presente estudio no han demostrado diferencias estadísticamente significativas en el rendimiento motor de los niños del grupo pies pronados respecto a los niños del grupo pies neutros, en los cinco ejercicios de resistencia de tipo aeróbicos evaluados en la investigación. 
Sin embargo, sí se han observado diferencias descriptivas entre ambos grupos, siendo estas repeticiones inferiores en el grupo pies pronados. Aunque no hubo significación en los cinco test realizados, los ejercicios de alternancia de piernas en banco sueco y salto a la comba se aproximaron a la significación estadística en el presente estudio.

\section{CONFLICTO DE INTERESES}

Los autores no presentan ningún tipo de conflicto de interés.

\section{FINANCIACIÓN}

Este trabajo de investigación no ha recibido financiación por parte de ninguna entidad.

\section{BIBLIOGRAFÍA}

1. Moreno de la Fuente JL. Podología general y biomecánica. 2. ed. Barcelona: Elsevier/Masson; 2009. p. 448.

2. Moreno de la Fuente JL. Podología Deportiva. 2. ed. Madrid: Masons; 2005. p. 416.

3. Rodriguez Valverde E. ¿Dolor de espalda y rodilla? ¡La respuesta está en los pies! United P.c. 2012. p. 242.

4. De la Rubia A. Lesiones del corredor : manual para prevenir y curar los problemas del running. Madrid: La esfera de los libros; 2017. p. 353.

5. Djaali W, Kusumaningtyas S, Ibrahim E, Sukur A, Mighra BA, Jakarta UN, et al. Analysis of energy expenditure during walking using the oxygen consumption method based on the arch type of the students at the sports science faculty of universitas Negeri Jakarta. Fac Ciencias del Deport Univ Negeri Jakarta. 2019. Disonible en: https://eudl.eu/ pdf/10.4108/eai.26-10-2018.2288917.

6. Lee HJ, Lim KB, Yoo JH, Yoon SW, Jeong TH. Effect of foot orthoses on children with lower extremity growing pains. Ann Rehabil Med. 2015;39(2):285-93. DOI: 10.5535/arm.2015.39.2.285.

7. Kumala MS, Tinduh D, Poerwandari D. Comparison of Lower Extremities Physical Performance on Male Young Adult Athletes with Normal Foot and Flatfoot. Phys Med Rehabil J Surabaya. 2019;1(1):6-13.

8. Roohi B, Hedayati S, Aghayari A. The effect of flexible flat-footedness on selected physical fitness factors in female students age 14 to 17 years. J Hum Sport Exerc. 2013;8(3):788-96. DOI: 10.4100/jhse.2013.83.03.

9. Chang-Ryeol L, Myung-Kwon K, Mi S. The Relationship between Balance and Foot Pressure in Fatigue of the Plantar Intrinsic Foot Muscles of Adults with Flexible Flatfoot. J Phys Ther Sci. 2012;24(8):699-701. DOI: 10.1589/jpts.24.699.

10. Tudor A, Ruzic L, Sestan B, Sirola L, Prpic T. Flat-footedness is not a disadvantage for athletic performance in children aged 11 to 15 years. Pediatrics. 2009;123(3):e386-92. DOI: 10.1542/peds.2008-2262.

11. Shih $\mathrm{Y}-\mathrm{F}$, Chen $\mathrm{C}-\mathrm{Y}, \mathrm{Chen} \mathrm{W}-\mathrm{Y}$, Lin $\mathrm{H}-\mathrm{C}$. Lower extremity kinematics in children with and without flexible flatfoot: a comparative study. BMC Musculoskelet Disord. 2012;13(1):31. DOI: 10.1186/1471-2474-13-31.
12. Arévalo-Mora JF, Reina-Bueno M, Munuera P V. Influence of Children's Foot Type on Their Physical Motor Performance. J Am Podiatr Med Assoc. 2016;106(1):15-21. DOI: 10.7547/14-086.

13. Delgado-Abellán L, Aguado X, Jiménez-Ormeño E, Mecerreyes L, Alegre L. Efectos del ejercicio continuo e intermitente sobre la huella plantar. Arch Med Deport. 2012;148:601-8.

14. Teyhen DS, Stoltenberg BE, Eckard TG, Doyle PM, Boland DM, Feldtmann JJ, et al. Static Foot Posture Associated With Dynamic Plantar Pressure Parameters. J Orthop Sport Phys Ther. 2011;41(2):100-7. DOI: 10.2519/jospt.2011.3412.

15. Berdejo del Fresno D, Lara Sánchez AJ, Martínez López EJ, Cachón Zagalaz J, Lara Diéguez S. Footprint modifications according to the physical activity practiced. Rev Int Med y Ciencias la Act Fis y del Deport. 2013;13(49):19-39.

16. Boozari S, Jamshidi AA, Sanjari MA, Jafari H. Effect of functional fatigue on vertical ground-reaction force in individuals with flat feet. J Sport Rehab. 2013;22(3):177-83. DOI: 10.1123/jsr.22.3.177.

17. Escamilla-Martínez E, Martínez-Nova A, Gómez-Martín B, Sánchez-Rodríguez R, Fernández-Seguín LM. The effect of moderate running on foot posture index and plantar pressure distribution in male recreational runners. J Am Podiatr Med Assoc. 2013;103(2):121.5. DOI: 10.7547/1030121.

18. Queen RM, Mall NA, Nunley JA, Chuckpaiwong B. Differences in plantar loading between flat and normal feet during different athletic tasks. Gait Posture. 2009;29(4):582-6. DOI: 10.1016/j.gaitpost.2008.12.010.

19. Anbarian M, Esmaeili $H$. Effects of running-induced fatigue on plantar pressure distribution in novice runners with different foot types. Gait Posture. 2016;48:52-6. DOI: 10.1016/j.gaitpost.2016.04.029.

20. Murley GS, Menz HB, Landorf KB. Foot posture influences the electromyographic activity of selected lower limb muscles during gait. J Foot Ankle Res. 2009;2(1):35. DOI: 10.1186/1757-1146-2-35.

21. Redmond AC, Crane YZ, Menz HB. Normative values for the Foot Posture Index.J Foot Ankle Res. 2008; 1(1):6. DOI: 10.1186/1757-1146-1-6.

22. Acerca del índice de masa corporal para niños y adolescentes | Peso Saludable | DNPAO | CDC [Internet]. [cited 2018 Dec 6]. Available from: https://www.cdc.gov/healthyweight/spanish/assessing/bmi/ childrens_bmi/acerca_indice_masa_corporal_ninos_adolescente.

23. Ministerio de sanidad servicios sociales e igualdad. Agencia Española de Consumo, Seguridad Alimentaria y Nutrición. Programa Perseo. [Internet]. Actualizada el 3 de febrero de 2014. [cited 2014 Oct 19]. Available from: http://www.perseo.aesan.msssi.gob.es/es/programa/ secciones/programa_perseo.shtml

24. Zurita Ortega F, Martínez Martínez A, Zurita Ortega A. Influencia de la tipología del pie en la actividad físico deportiva. Fisioterapia. 2007;29(2):74-9. DOI: 10.1016/S0211-5638(07)74417-6.

25. Kaufman K, Brodine S, Shaffer R, Johnson C, Cullison T. The effect of foot structure and range of motion on musculoskeletal overuse injuries. Am J Sport Med. 1999;27(5):585-93. DOI: 10.1177/03635465990270050701.

26. Mora J. Teoría del entrenamiento y del acondicionamiento físico. Cádiz: COPLEF Andalucía; 1995. p. 412.

27. Lin CJ, Lai KA, Kuan TS, Chou YL. Correlating factors and clinical significance of flexible flatfoot in preschool children. J Pediatr Orthop. 2001;21(3):378-82. 